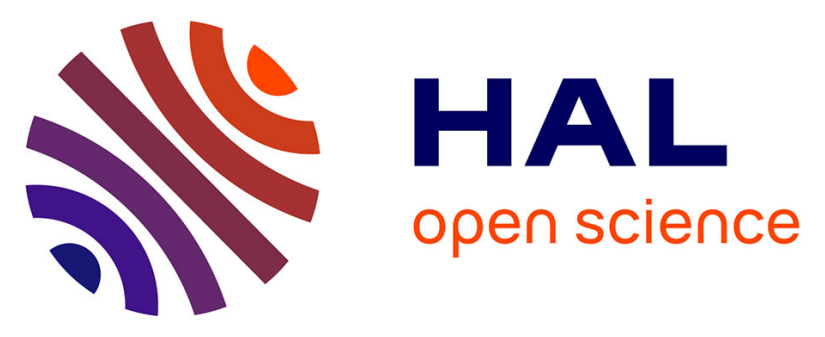

\title{
Wavelet-based Multifractal Analysis of Heartbeat Dynamics: Non-Gaussian Expansion vs. Cumulants
}

Vincenzo Catrambone, Herwig Wendt, Riccardo Barbieri, Patrice Abry, Gaetano Valenza

\section{- To cite this version:}

Vincenzo Catrambone, Herwig Wendt, Riccardo Barbieri, Patrice Abry, Gaetano Valenza. Waveletbased Multifractal Analysis of Heartbeat Dynamics: Non-Gaussian Expansion vs. Cumulants. 11th Conference of the European Study Group on Cardiovascular Oscillations (ESGCO 2020), Jul 2020, Pisa, Italy. 10.1109/ESGCO49734.2020.9158011 . hal-03034103

\section{HAL Id: hal-03034103 \\ https://cnrs.hal.science/hal-03034103}

Submitted on 1 Dec 2020

HAL is a multi-disciplinary open access archive for the deposit and dissemination of scientific research documents, whether they are published or not. The documents may come from teaching and research institutions in France or abroad, or from public or private research centers.
L'archive ouverte pluridisciplinaire HAL, est destinée au dépôt et à la diffusion de documents scientifiques de niveau recherche, publiés ou non, émanant des établissements d'enseignement et de recherche français ou étrangers, des laboratoires publics ou privés. 


\title{
Wavelet-based Multifractal Analysis of Heartbeat Dynamics: Non-Gaussian Expansion vs. Cumulants
}

\author{
V. Catrambone ${ }^{1 *}$, H. Wendt ${ }^{2}$, R. Barbieri ${ }^{3}$, P. Abry ${ }^{4}$, and G. Valenza ${ }^{1}$
}

\begin{abstract}
Nonlinear and non-Gaussian analysis contributes to a comprehensive characterization of autonomic nervous system control on heartbeat dynamics. Nevertheless, a statistical comparison between non-Gaussian features and cumulants in the frame of a wavelet-based multifractal analysis of heartbeat dynamics has not been performed yet. Here we exploit a multifractal features formulation based on wavelet p-leaders spectrum applied to instantaneous heartbeat estimates from inhomogeneous point processes. We then perform a non-Gaussian multiscale expansion and analyze physiologically-meaningful differences between resting state and cold-pressure test in 30 healthy subjects. Results show that nonlinear and non-Gaussian features are associated with statistical differences between physiological states, whereas cumulants from the multifractal spectrum, till the third order, were not statistically different.
\end{abstract}

\section{INTRODUCTION}

Cardiovascular dynamics result from a continuous interplay between different physiological systems occurring at an anatomical and functional levels [1]. This interplay is thought to be responsible for generating non-stationary and nonlinear characteristics in heartbeat dynamics as quantified from Heart Rate Variability (HRV) series [1]. To this end, exemplary fractal, multiscale, and non-Gaussian analysis has been successfully employed for the characterization of autonomic control on heartbeat in health and disease [1]-[3].

Cardiovascular dynamics does not show a self-similar dynamics because different Hurst exponents exist at different time scales. Hence, multifractal (MF) spectra can be used for a comprehensive characterization of heartbeat dynamics detecting fluctuations in self-regularity. Methods including detrended fluctuation analysis and modulus maxima method of the wavelet transform were proposed to perform a MF analysis. Recently, a methodological framework quantifying nonlinear and non-Gaussian features through the p-leaders generalization of the wavelet leaders MF formalism has been proposed [4], [5].

Although a multiscale MF analysis has recently been proven effective when integrated with inhomogeneous pointprocess modelling for heartbeat dynamics [2], [6], a statistical comparison on p-leaders-derived features from a nonGaussian expansion and cumulants has not been performed

\footnotetext{
${ }^{1}$ Bioengineering and Robotics Research Center E.Piaggio \& Dept. of Information Engineering, University of Pisa, Pisa, Italy;

2 IRIT, Université de Toulouse, CNRS, France;

${ }^{3}$ Department of Electronics, Informatics and Bioengineering, Politecnico di Milano, Italy;

${ }^{4}$ Univ Lyon, ENS de Lyon, Univ Claude Bernard, CNRS, Laboratoire de Physique, Lyon, France;

* Corresponding author. Email at: vincenzo.catrambone@ing.unipi.it;

Work supported in part by ANR-16-CE33-0020 MultiFracs, France
}

yet. Therefore, in this study we investigate MF cumulants and features from a non-Gaussian expansion, from instantaneous cardiovascular dynamics while discerning between different sympathovagal changes. Particularly, we process cardiovascular data from healthy subjects undergoing a cold-pressor test (CPT), which provides a significant thermal peripheral elicitation and activates the autonomic nervous system [7][9].

\section{Materials AND Methods}

\section{A. Experimental Setup}

Details on the experimental procedure are reported in [6], [7]. Briefly, thirty right-handed healthy volunteers (15 females, average age of 26.7 years) sat comfortably on a chair and underwent a 3-minute resting state and a subsequent CPT for up to 3 minutes. Data from six subjects were not considered for further analyses because of corrupted recordings. One-lead ECG was recorded continuously with a $500 \mathrm{~Hz}$ sampling rate, and R-peaks were automatically detected using the Pan-Tompkin's algorithm. RR interval series were then processed by using inhomogeneous pointprocess models for heartbeat dynamics [10], from which we derived instantaneous estimates of first-order moment (mean-HR) of the probability density function predicting each heartbeat interval [10].

\section{B. Multifractals and non-Gaussian Multiscale analyses}

The series self-similarity can be formalized through the wavelet spectrum, $S_{d_{X}}$, modelled through a power law whose exponent is controlled by the Hurst parameter $H$ and the well-known discrete wavelet transform $d_{X}$ [11].

Multifractality extends the self-similarity concept by introducing the temporal variability of the Hurst exponent $H=h(t)$, such that the model can be described through a multifractal spectrum $D(h)$. This spectrum collects the temporal repetition of the level series of $h(t)$ [5]. The quantification of $D(h)$ requires a range of moments $q$ and wavelet p-leaders $\ell_{X}^{(p)}$ defined as the local $\ell^{p}$ norms of wavelet coefficients within a thin temporal neighbourhood over all finer scales. Peculiar properties of $D(h)$ can be estimated through the use of its cumulants:

$$
C_{m}^{(p)}(j) \equiv \operatorname{Cum}_{m} \log \left(\ell_{X}^{(p)}(j)\right) \simeq c_{m}^{0}+c_{m} \log \left(2^{j}\right)
$$

where mode, width, asymmetry, and flatness of $D(h)$ are quantified by $c_{1}, c_{2}, c_{3}$ and $c_{4}$, respectively [5].

Starting from (1), a recently proposed multiscale nonGaussian expansion can be defined as follows [4]: 


$$
L_{q}^{2 P}(j)=\sum_{m=2}^{\infty} C_{m}(j) \frac{\sum_{i=1}^{P} q_{2 i-1}^{m-1}-q_{2 i}^{m-1}}{m !}
$$

It has been proven that higher-order cumulants, and thus non-Gaussian properties, can be estimated through the $L_{q}^{2 P}(j)$ coefficients. The sensitivity of $L_{q}^{2 P}(j)$ to different kinds of departure from Gaussianity can be tuned by the choice of the moments order $q_{i}$ [4]. Note that $L_{q}^{2 P}(j)$ quantifies only nonlinear data properties, as $C_{1}(j)$ is not in (2).

\section{Statistical Analysis AND Results}

We investigated statistical differences between rest and CPT at different time scales using $L_{q}^{2 P}(s)$ coefficients gathered from a non-Gaussian multiscale expansion of meanHR. Features including LQ1, LQ2, and LQ3 are listed in Table I. The multifractal analysis is implemented by employing a Daubechies wavelet function, with $N_{\phi}=3$ vanishing moment. Statistical differences were investigated using Wilcoxon non-parametric tests for paired samples.

Results show that cumulants derived from the mean-HR multifractal spectrum are not associated with significant differences (see Figure 1), whereas indices from the nonGaussian expansion are associated with significant differences at different time scales (see Figure 2).

TABLE I: Non-Gaussian Expansion Indices

\begin{tabular}{c|cr}
\hline & moments $q_{i}$ & cumulants $C_{m}$ active in (2) \\
\hline LQ1 & $(0.25,2)$ & $m \geq 2$ \\
LQ2 & $(-2,2)$ & any departure from Gaussian \\
& $m=2,4, \ldots$ \\
LQ3 & $(0.25,0.75,2.5,2))$ & symmetric properties \\
$m \geq 3$ \\
\end{tabular}
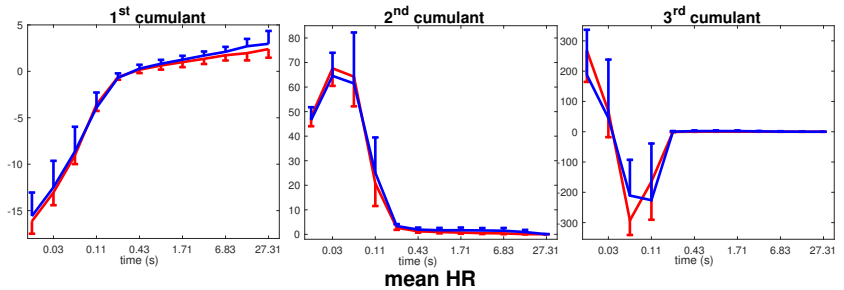

Fig. 1: First three order cumulants calculated from mean-HR of a point-process model during resting (blue) and CPT (red) sessions. Values indicate the median across subjects, whereas vertical bars refer to the median absolute deviation. Asterisks indicate statistically significant differences between sessions $(p<0.05)$.

\section{Discussion AND CONCLUSION}

In this study we characterized the multifractal behaviour of heartbeat dynamics during a CPT elicitation with respect to a resting state in healthy subjects. We exploited the recently defined wavelet-based multifractal analysis in cardiovascular dynamics derived from inhomogeneous point-process models [10] and investigated statistical differences between sessions

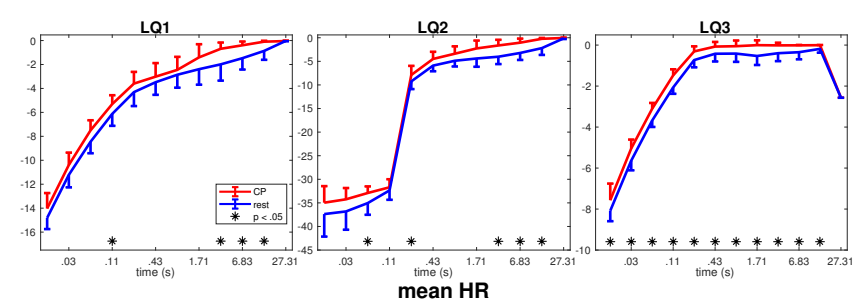

Fig. 2: LQ1, LQ2, LQ3 calculated from mean-HR of a point-process model during resting (blue) and CPT (red) sessions. Values indicate the median across subjects, whereas vertical bars refer to the median absolute deviation. Asterisks indicate statistically significant differences between sessions $(p<0.05)$.

using features from a non-Gaussian expansion and cumulants.

We demonstrated that non-Gaussian indices derived from cardiac dynamics tend to a zero value at higher scales, thus depicting a progression towards gaussianity from lower scales to higher scales. Moreover, results are in agreement with [6] showing a general higher non-Gaussianity during the resting phases with respect to a CPT. Interestingly, major differences are with LQ3, which quantifies the belonging to a non-log-normal distribution. We conclude that cardiovascular dynamics have an inherent non-Gaussianity decreasing trend when a haemodynamic stress is applied (i.e. CPT cause an increased mean arterial and blood pressure [8]), differently from cumulants. Other variables from the point process framework will be investigated in future studies using different heartbeat datasets.

\section{REFERENCES}

[1] U. Acharya et al., "Heart rate variability," in Advances in cardiac signal processing, pp. 121-165, Springer, 2007.

[2] G. Valenza et al., "Mortality prediction in severe congestive heart failure patients with multifractal point-process modeling of heartbeat dynamics," IEEE Transactions on Biomedical Engineering, 2018.

[3] P. C. Ivanov, L. A. N. Amaral, A. L. Goldberger, S. Havlin, M. G. Rosenblum, Z. R. Struzik, and H. E. Stanley, "Multifractality in human heartbeat dynamics," Nature, vol. 399, no. 6735, p. 461, 1999.

[4] H. Wendt et al., "Wavelet $p$-leader non gaussian multiscale expansions for heart rate variability analysis in congestive heart failure patients," IEEE Trans. on Biomedical Engineering, vol. 66(1), pp. 80-88, 2019.

[5] H. Wendt et al., "Bootstrap for empirical multifractal analysis," IEEE signal processing magazine, vol. 24, no. 4, pp. 38-48, 2007.

[6] V. Catrambone et al., "Heartbeat dynamics analysis under coldpressure test using wavelet p-leader non-gaussian multiscale expansions," in Engineering in Medicine and Biology Society (EMBC), 2019 41st Annual International Conference of the IEEE, IEEE, 2019.

[7] V. Catrambone et al., "Time-resolved directional brainheart interplay measurement through synthetic data generation models," Annals of Biomedical Engineering, 2019.

[8] S. O. Elias and R. E. Ajayi, "Effect of sympathetic autonomic stress from the cold pressor test on left ventricular function in young healthy adults," Physiological reports, vol. 7, no. 2, p. e13985, 2019.

[9] J. Cui et al., "Baroreflex modulation of muscle sympathetic nerve activity during cold pressor test in humans," Am. J. of Phys, vol. 282, no. 5, pp. H1717-H1723, 2002.

[10] G. Valenza, L. Citi, E. P. Scilingo, and R. Barbieri, "Point-process nonlinear models with laguerre and volterra expansions: Instantaneous assessment of heartbeat dynamics," IEEE Transactions on Signal Processing, vol. 61, no. 11, pp. 2914-2926, 2013.

[11] S. Mallat, A wavelet tour of signal processing. Elsevier, 1999. 